\title{
Cirurgia aórtica totalmente laparoscópica para tratamento de isquemia crítica de membros: relato do primeiro caso no Brasil
}

\author{
Total laparoscopic aortic surgery for treatment of limb critical \\ ischemia: report of the first case in Brazil \\ Ricardo de Alvarenga Yoshida ${ }^{1}$, Winston Bonetti Yoshida ${ }^{2}$, Hamilton de Almeida Rollo ${ }^{2}$, \\ Ralf Kolvenbach $^{3}$, Rodrigo Gibin Jaldim ${ }^{4}$, Fernando Cordeiro Pimentel ${ }^{4}$, \\ Aline Helena Gonzáles Fares ${ }^{4}$
}

\begin{abstract}
Resumo
A cirurgia videolaparoscópica (CVL) vem evoluindo como alternativa cirúrgica menos invasiva para o tratamento da doença aterosclerótica oclusiva aortoilíaca. O objetivo deste relato de caso foi demonstrar os resultados da primeira cirurgia aórtica totalmente laparoscópica relatada no Brasil para o tratamento da doença oclusiva aortoilíaca em paciente com isquemia crítica. Os tempos cirúrgicos totais de dissecção e exposição da aorta antes do clampeamento, exposição retroperitoneal da aorta, clampeamento total e da anastomose proximal com técnica totalmente laparoscópica foram de 220 minutos, 15 e 27 minutos, 42 minutos, 110 minutos e 78 minutos, respectivamente. A técnica videolaparoscópica é mais uma ferramenta minimamente invasiva, viável, segura e eficaz para o tratamento da doença oclusiva aortoilíaca extensa. Ela, que nada mais é do que a cirurgia convencional realizada sob visão laparoscópica, tem bons resultados a longo prazo, que se associam à elegância técnica.
\end{abstract}

Palavras-chave: Cirurgia videoassistida, aorta, doenças vasculares, aterosclerose.

\section{Introdução}

A aterosclerose é tida como uma doença da civilização, que cresce com ela e que a cada dia acomete indivíduos mais jovens ${ }^{1-3}$. De distribuição mundial aparentemente regular, manifesta-se em cerca de 3 a 10\% da população, aumentando para 15 a $20 \%$ em pessoas acima de 70 $\operatorname{anos}^{1-3}$. A incidência média anual de doença arterial obstrutiva periférica (DAOP) ou aterosclerose sintomática, de acordo com estudo de Framingham ${ }^{4}$, é de 26 por 10.000

\begin{abstract}
Laparoscopic surgery has been increasingly used as a less invasive surgical option for the treatment of aortoiliac occlusive atherosclerotic disease. The objective of this case report was to describe the results of the first total laparoscopic surgery conducted in Brazil for the treatment of aortoiliac occlusive disease in a patient with critical ischemia. Total time of surgery, time of aortic dissection and exposure before clamping, time of retroperitoneal exposure of the aorta, and time of total clamping and proximal anastomosis using total laparoscopic technique were $220 \mathrm{~min}$ utes, 15 and 27 minutes, 42 minutes, 110 minutes, and 78 minutes, respectively. The laparoscopic technique is an additional minimally invasive tool, being feasible, safe, and effective for the treatment of extensive aortoiliac occlusive disease. This technique, which is nothing more than a conventional surgery performed under laparoscopic viewing, brings good long-term results associated with technical elegance.
\end{abstract}

Keywords: Video-assisted surgery, aorta, vascular diseases, atherosclerosis.

homens e de 12 por 10.000 mulheres, aumentado com a idade.

Dos pacientes com 50 anos ou mais, na apresentação inicial desta doença, 20 a 30\% encontram-se assintomáticos, 30 a $40 \%$ têm dores atípicas nas pernas, 10 a 35\% têm claudicação intermitente típica e 1 a $3 \%$ têm isquemia crítica $^{5}$. Após 5 anos de evolução, dos pacientes que não se apresentam inicialmente com isquemia crítica, 70 a $80 \%$ estabilizam os sintomas de claudicação, 10 a $20 \%$ pioram

\footnotetext{
1. Cirurgião vascular e endovascular. Doutorando e colaborador, Disciplina de Cirurgia Vascular e Endovascular, Faculdade de Medicina de Botucatu (FMB), UNESP, Botucatu, SP.

2. Professor adjunto livre-docente, Disciplina de Cirurgia Vascular e Endovascular, FMB, UNESP, Botucatu, SP.

3. Professor titular. Chefe do Serviço de Cirurgia Vascular e Endovascular, Hospital Augusta, Dusseldorf, Alemanha.

4. Residente, Disciplina de Cirurgia Vascular e Endovascular, FMB, UNESP, Botucatu, SP.

Os autores declaram não possuir interesses comerciais nos produtos usados neste trabalho. Entretanto, receberam apoio das empresas Johnson \& Johnson e Karl Storz.

Artigo submetido em 08.06.09, aceito em 05.10.09.
}

J Vasc Bras. 2010;9(1):66-73.

Copyright (C 2010 by Sociedade Brasileira de Angiologia e de Cirurgia Vascular 
da claudicação e 5 a $10 \%$ desenvolvem isquemia crítica ${ }^{5}$. Já dos pacientes que se apresentam inicialmente com isquemia crítica, $45 \%$ permanecem vivos com os dois membros, $30 \%$ são amputados e $25 \%$ falecem ${ }^{5,6}$.

Em geral, as isquemias críticas por oclusões aortoilíacas são mais graves quando apresentam oclusão completa desse território (lesões TASC D) ou quando associadas a lesões subjacentes do território femoropoplíteo ou poplíteo-distal ${ }^{5}$. O padrão-ouro para o tratamento das lesões TASC D é a cirurgia convencional, incluindo: enxertos anatômicos (trasperitoneais ou retroperitoneais), enxertos extra-anatômicos e endarterectomias; porém, a técnica endovascular pode ser empregada para casos selecionados ${ }^{5}$. Mais recentemente têm sido relatadas experiências com a cirurgia videoassistida para esses casos vista como uma alternativa minimante invasiva para as reconstruções aortoilíacas $^{7-10}$.

O objetivo deste relato de caso é demonstrar os resultados da primeira cirurgia totalmente laparoscópica relatada no Brasil para o tratamento da doença oclusiva aortoilíaca em paciente com isquemia crítica.

\section{Relato do caso}

Paciente do sexo masculino, lavrador, 57 anos, proveniente de Igaraçu do Tietê (SP), apresentava história de claudicação intermitente para $500 \mathrm{~m}$ na panturrilha esquerda há 3 anos com piora progressiva até que, 30 dias antes da internação, apresentava dor mesmo sem andar (dor em repouso), esfriamento do pé esquerdo e arroxeamento local. Para aliviar a dor necessitava deixar o membro pendente e fazer uso de analgésicos fortes. A arteriografia (23/04/2009) mostrou oclusão da artéria ilíaca externa esquerda com reenchimento da artéria femoral comum esquerda e de seus ramos (Figura 1). A ultrassonografia (US) com Doppler colorido (duplex) mostrou ausência de aneurismas e pouca calcificação arterial (6/5/09) (Figura 2). O paciente apresentava como comorbidades associadas: hipertensão arterial sistêmica (HAS), tabagismo (uso de fumo de corda por 17 anos) e ex-etilismo.

Ao exame físico, o paciente apresentava cianose e palidez no pé esquerdo e não apresentava qualquer pulso palpável no membro inferior esquerdo (MIE), sendo que à direita podiam ser palpados todos os pulsos com intensidade diminuída, principalmente no caso dos distais. O índice tornozelo-braquial (ITB) mostrou os seguintes valores

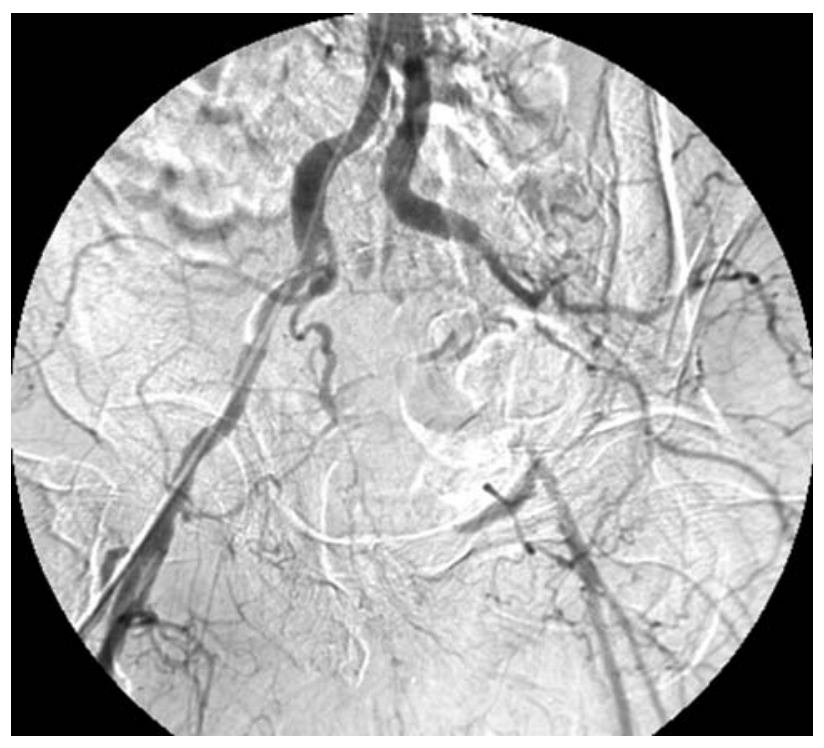

Figura 1 - Arteriografia: oclusão da artéria ilíaca externa esquerda com reenchimento da artéria femoral comum esquerda e de seus ramos

para o MIE: poplíteo $(\mathrm{POP})=0,62$; tibial anterior $(\mathrm{TA})=0,41$; tibial posterior $(\mathrm{TP})=0,45$ e fibular $(\mathrm{Fib})=0,45$.

Exames pré-operatórios e avaliação cardiológica padrão revelaram risco intermediário para evento cardiovascular, segundo o algoritmo do American College of Physicians.

O objetivo foi fazer a cirurgia de reconstrução aortoilíaca por meio de enxerto aortofemoral por técnica totalmente laparoscópica. O paciente foi submetido a anestesia geral e introdução de sonda nasogástrica. A seguir, o paciente foi posicionado na mesa operatória sobre um coxim, em decúbito lateral direito, com o braço esquerdo acima do tronco (Figura 3 A). Após antissepsia e colocação de campos estéreis, iniciou-se a operação com dissecção da artéria femoral e de seus ramos na virilha. A seguir, foi introduzido trocar ao lado da cicatriz umbilical, iniciando-se o pneumoperitônio por essa via. Este foi mantido com pressão de $12 \mathrm{~mm} \mathrm{Hg}$ de $\mathrm{CO}_{2}$. Pelo trocar, foi introduzida uma ótica de $30^{\circ}$. Dois outros trocares de $11 \mathrm{~mm}\left(\right.$ Endopath $^{\circledR}$ Johnson \& Johnson) foram colocados lateralmente à linha média, abaixo e acima do umbigo (Figura 3 B). Outros quatro trocares foram colocados em torno do lado esquerdo, tendo como referência o rebordo costal, a linha axilar média, musculatura dorsal e crista ilíaca, totalizando sete portais (Figura 4). A exposição da aorta iniciou-se com a 

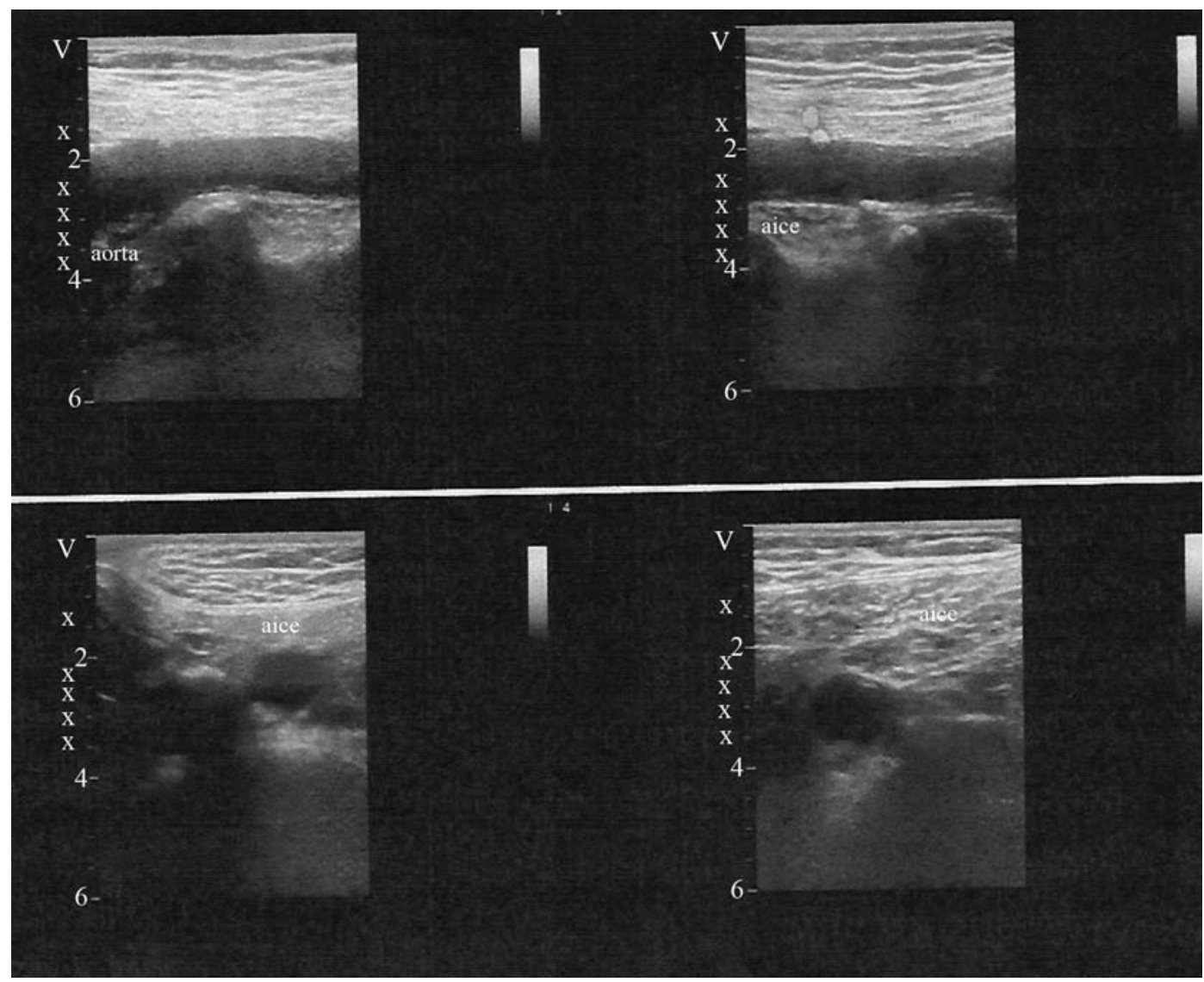

Figura 2 - Resultado do duplex - ausência de aneurismas e pouca calcificação arterial
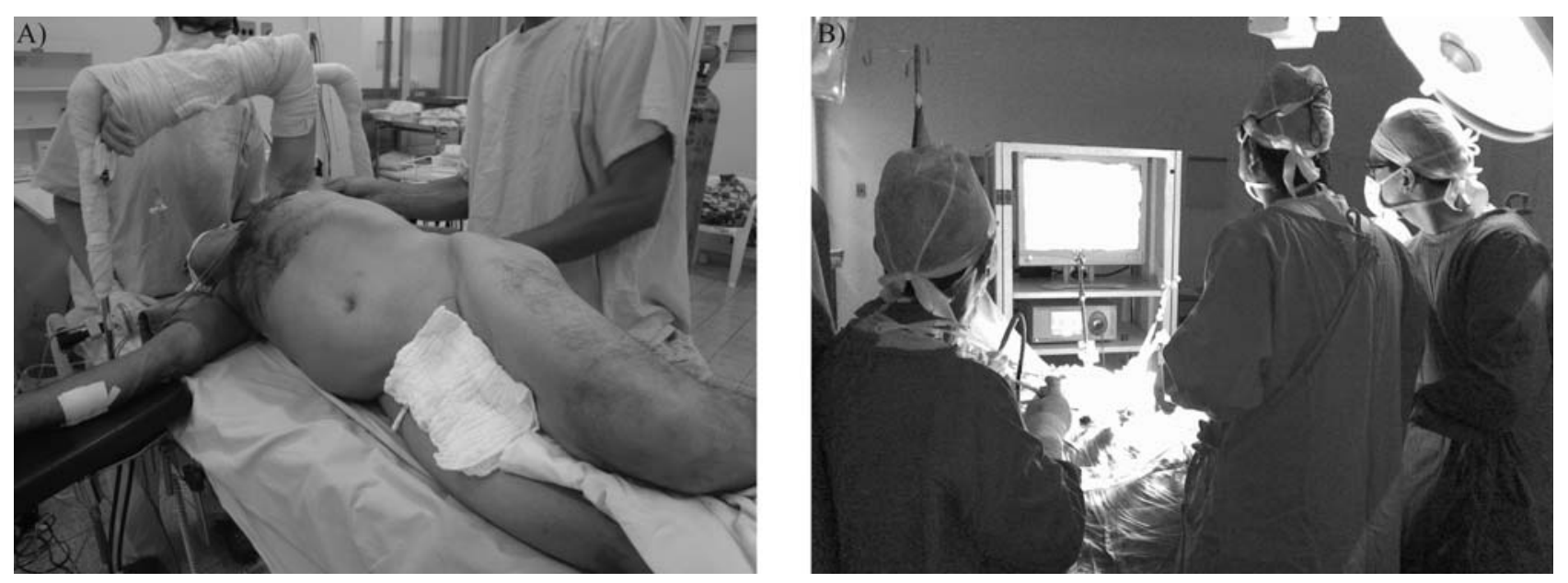

Figura 3 - A) Posicionamento do paciente na mesa operatória em sobre um coxim, em decúbito lateral direito, com o braço esquerdo acima do tronco; B) cirurgia totalmente laparoscópica

mobilização medial do hemicólon esquerdo distal usando graspers, tesouras laparoscópicas e bisturi harmônico (ULTRACISION $^{\circledR}$ - Johnson \& Johnson), seguindo as orientações da técnica Apron ${ }^{11}$ (Figura 5). Após a completa dissecção da aorta distal e da sua bifurcação (Figura 5), foi administrada heparina endovenosa (100 UI/kg). Um clampe aórtico laparoscópico (clampe vascular laparoscópico Storz $^{\circledR}$ ) foi posicionado na aorta distal infrarrenal, e o ou- 


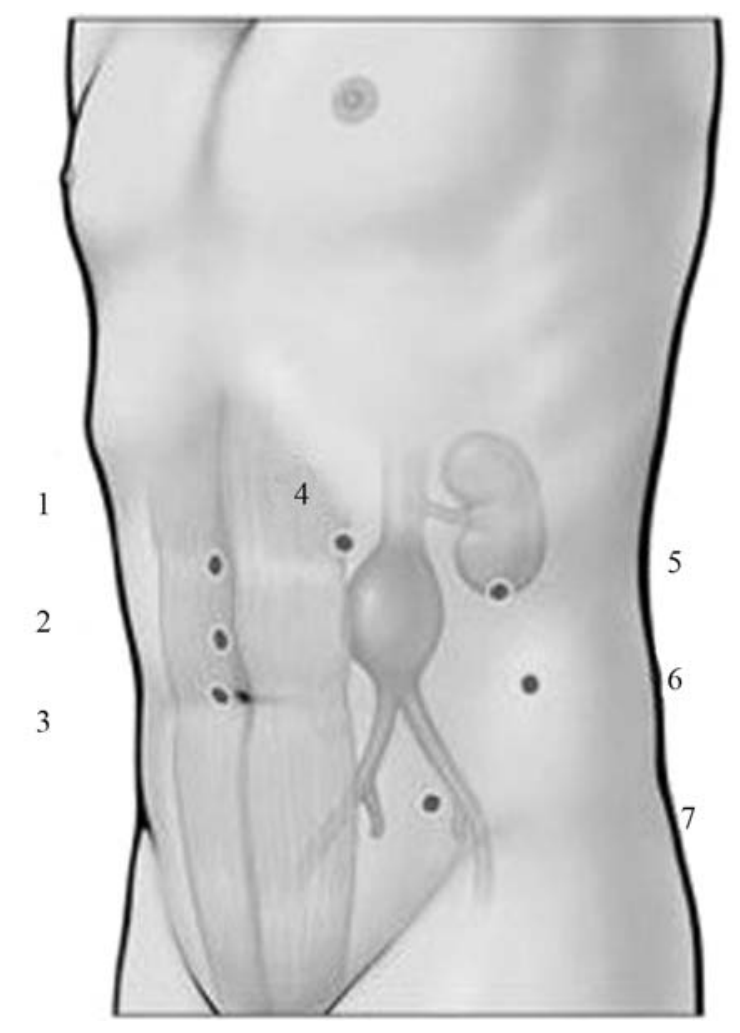

Figura 4 - Posição esquemática dos trocares

tro clampe, na aorta logo acima de sua bifurcação através de portais localizados abaixo do rebordo costal e crista ilíaca, respectivamente (Figura 6). Foi inserida prótese de Dacron $8 \mathrm{~mm}$ na cavidade através de um dos portais. Não foi necessária a ligadura de artérias lombares nesse segmento aórtico. Após aortotomia longitudinal, anastomose proximal foi realizada sob visão totalmente laparoscópica, utilizando-se técnica de Coggia $^{12}$ com sutura contínua com três fios Prolene ${ }^{\circledR} 4.0$ (Ethicon ${ }^{\circledR}$ - Johnson \& Johnson) entre a aorta distal e a prótese de Dacron 8 (Figura 7). Após liberação do fluxo e revisão de hemostasia, que não mostrou sangramentos pela sutura, a anastomose foi coberta por Surgicell $^{\circledR}$ (Ethicon $^{\circledR}$ - Johnson \& Johnson) e o enxerto foi recoberto pelo hemicólon $\mathrm{E}$ e pelo peritônio simplesmente recolocando o paciente em decúbito horizontal. Foi exteriorizado o ramo distal da prótese pela inguinotomia esquerda, previamente realizada no início da cirurgia, e feito fechamento dos portais. Foi deixado dreno de aspiração previsto para permanecer por até 24 horas.

Foi feita arteriotomia longitudinal da artéria femoral comum (AFC) esquerda, previamente exposta, notando-se refluxo normal pela artéria femoral profunda e nenhum re-

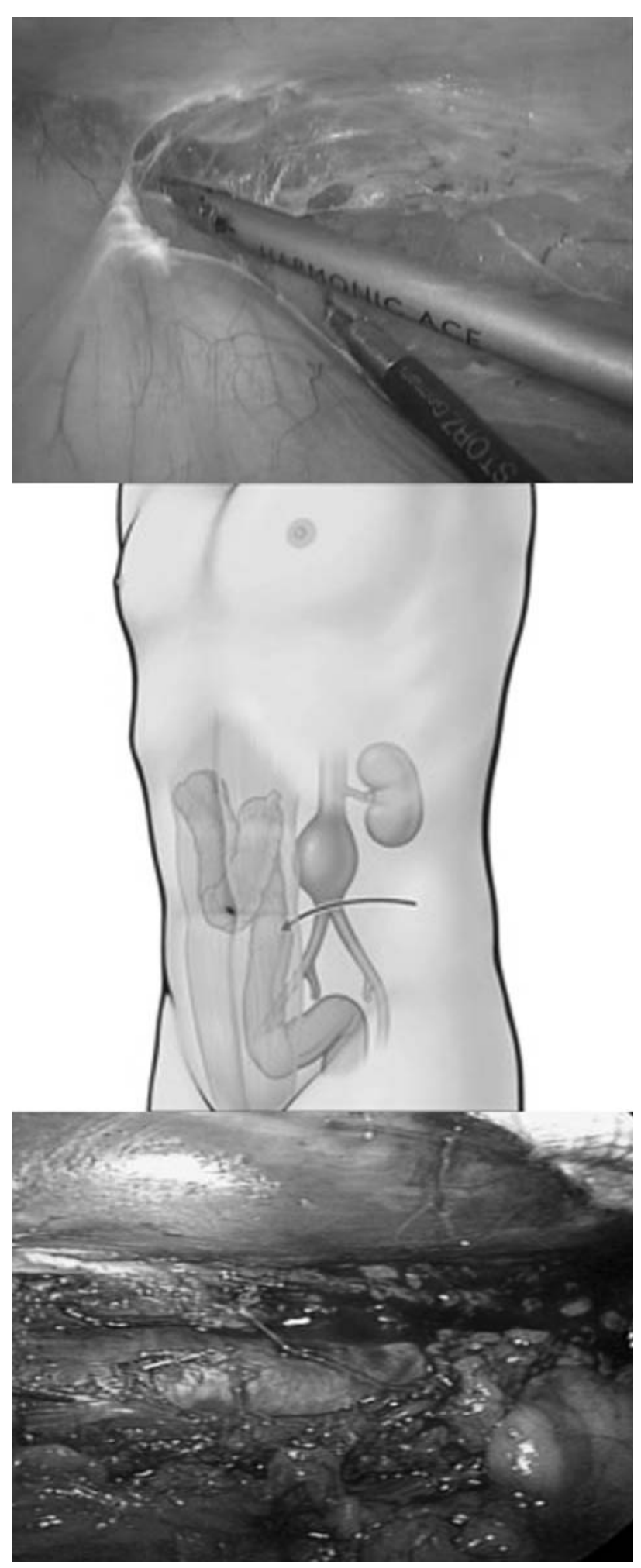

Figura 5 - Exposição da aorta com a mobilização medial do hemicólon esquerdo seguindo as orientações da técnica Apron ${ }^{11}$

fluxo pela artéria femoral superficial (AFS). Além disso, foi observada placa aterosclerótica ocupando grande parte 


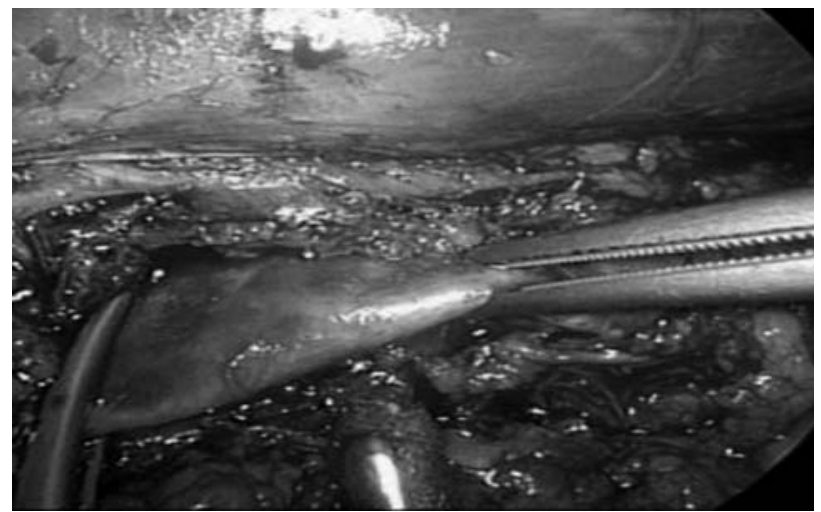

Figura 6 - Clampeamento vascular laparoscópico

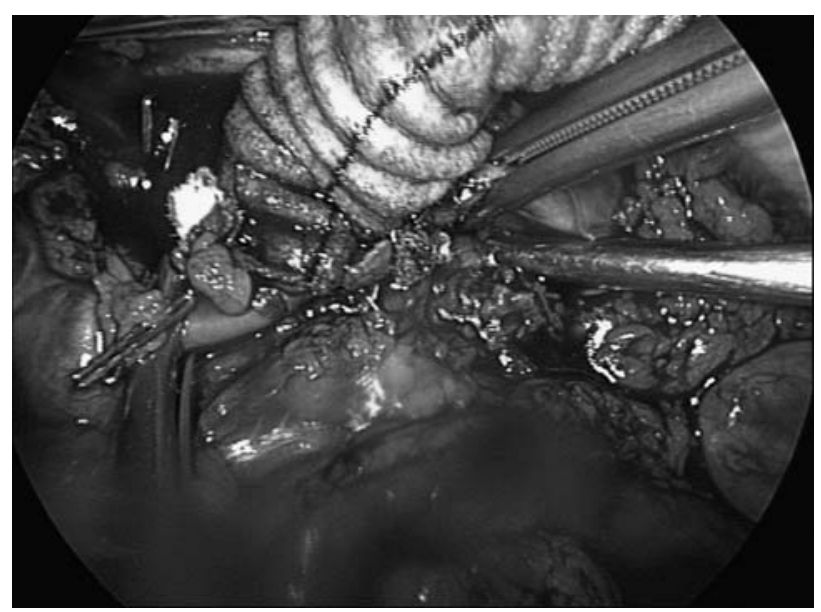

Figura 7 - Anastomose proximal realizada sob visão totalmente laparoscópica

da luz distal da AFC que se entendia para a AFS. Optou-se por endarterectomia da AFC distal e da AFS ostial, realizada com sucesso. Foi então confeccionada a anastomose término-lateral do enxerto na AFC (Figura 8). Ao final do procedimento, pulsos femoral, poplíteo e tibial anterior foram palpados.

O paciente evoluiu bem, com manutenção dos pulsos femoral, poplíteo e tibial anterior, com ITB à esquerda: $\mathrm{TP}=1,0 ; \mathrm{Fib}=1,0$. Apresentou dor no flanco esquerdo com peritonismo local. $\mathrm{O}$ hemiabdome direito não apresentava dor à palpação. Os ruídos hidroaéreos encontravam-se presentes, porém diminuídos. Os sinais vitais e a diurese estavam normais.Não houve alteração dos níveis de hematócrito e hemoglobina em relação ao pré-operatório. $\mathrm{O}$ paciente não apresentou náuseas, vômitos ou mal-estar. Foi submetido a tomografia computadorizada de controle, que revelou enxerto sem alterações, ausência

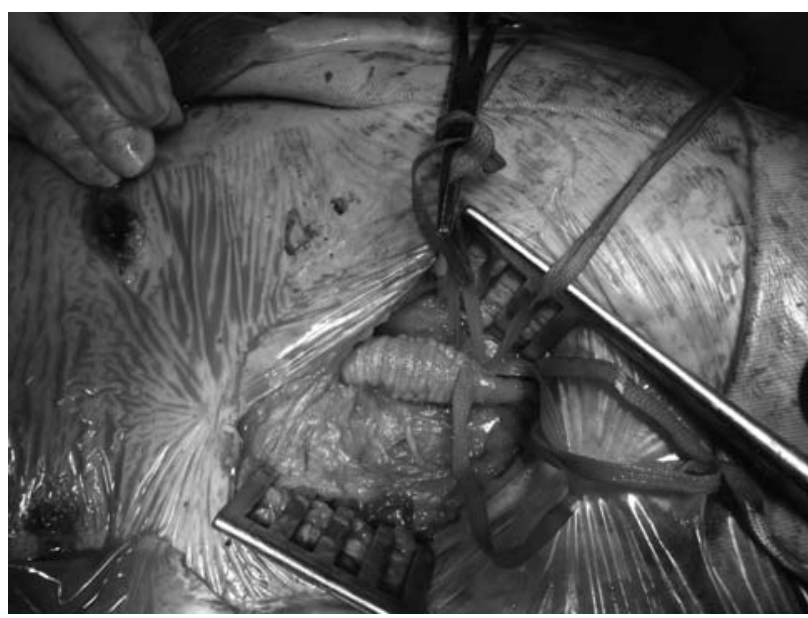

Figura 8 - Anastomose distal convencional término-lateral do enxerto na artéria femoral comum

de sangramentos, porém com pequeno hematoma local em hemiabdome esquerdo, não havendo sinais de alterações viscerais. A dor abdominal melhorou com dimeticona VO.

No segundo dia de pós-operatório (PO), o paciente encontrava-se praticamente sem dor abdominal, com todas as incisões sem alterações (Figura 9), alimentando-se re-

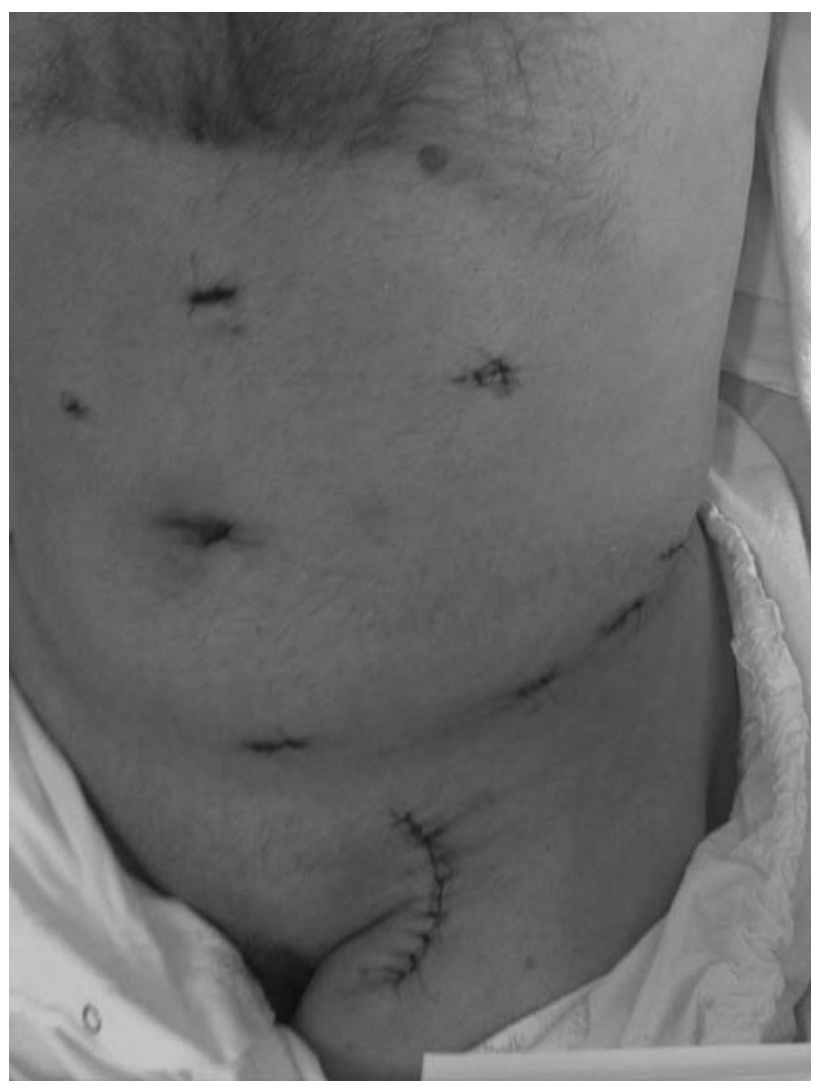

Figura 9 - Ferida cirúrgica no pós-operatório precoce 
gularmente e sem dor no membro operado. Teve alta no terceiro dia de PO, deambulando e sem queixas.

\section{Resultados}

O tempo cirúrgico total foi de 220 minutos. Os tempos de dissecção e exposição da aorta, antes do clampeamento, foram de 15 e 27 minutos, respectivamente. O tempo cirúrgico para a exposição retroperitoneal da aorta, antes do clampeamento aórtico, ambos realizados com a técnica Apron $^{11}$, bem como para sua dissecção com bisturi harmônico (ULTRACISION ${ }^{\circledR}$ ), foi de 42 minutos. O tempo de clampeamento total foi de 110 minutos, e o da anastomose proximal com técnica totalmente laparoscópica foi de 78 minutos.

Não houve lesões inadvertidas de vasos sanguíneos ou de estruturas vizinhas. A anastomose proximal foi revisada ao término da anastomose distal, não sendo evidenciados defeitos técnicos, estenoses ou vazamentos.

O inventário final da cavidade abdominal não mostrou lesões de órgãos intracavitários.

\section{Discussão}

A determinação do melhor método de revascularização para o tratamento sintomático da DAOP se baseia no equilíbrio entre o risco de uma intervenção específica e o grau de durabilidade da melhora que se pode esperar da mesma $^{5}$. Fluxo adequado e apropriado deságue são necessários para manter o funcionamento dos segmentos revascularizados $^{5}$. Em geral, os resultados da revascularização dependem da extensão da doença arterial subjacente, do grau da doença sistêmica (comorbidades que podem influenciar na sobrevida do paciente e na perviedade do enxerto) e do tipo de cirurgia realizada ${ }^{5}$.

Devido à gravidade dos pacientes portadores de DAOP, que apresentam doença sistêmica e multissegmentar acompanhada de inúmeras comorbidades ${ }^{13}$, houve sempre uma busca constante por procedimentos menos invasivos no tratamento cirúrgico desses pacientes, mas que mantivessem os bons resultados a longo prazo dos tratamentos convencionais. Dessa perspectiva, o tratamento endovascular trouxe considerável avanço para esses casos, com o advento das angioplastias com balão, implantes de stents e endopróteses, trombólises e aterectomias, dentre outros procedimentos ${ }^{5}$. Segundo o Inter-Society Consensus for the Management of Peripheral Arterial Disease
(TASC II) ${ }^{5}$, as lesões TASC A e B são as que apresentam os melhores resultados com o tratamento endovascular, sendo empregado este, então, como primeira escolha. Já as lesões TASC C e D podem ser tratadas pela técnica endovascular, mas seu uso só é justificado em pacientes que não toleram clinicamente a cirurgia convencional ${ }^{5}$.

Os acessos cirúrgicos mais utilizados para as reconstruções aortoilíacas são o transperitoneal e o retroperitone$\mathrm{al}^{14}$. Os dados da literatura não são uniformes quanto aos resultados comparativos entre essas duas abordagens, mas, em geral, em relação ao tempo cirúrgico, reposição de líquidos e hospitalização, são menores para a via retroperitoneal $^{14,15}$. De qualquer maneira, ambos os acessos são considerados procedimentos invasivos que necessitam de cuidados pós-operatórios intensivos, apresentando morbidade considerável, além de provocarem elevada resposta inflamatória e estresse oxidativo ${ }^{16,17}$.

A cirurgia videolaparoscópica tem sido empregada rotineiramente para o tratamento de oclusões aortoilíacas em alguns centros da Europa e do Canadá ${ }^{7-9,12,18}$. As vantagens apresentadas por esta técnica nas reconstruções aórticas são: menor invasividade, trauma cirúrgico reduzido, menor incisão abdominal e dor pós-operatória, alimentação oral precoce e permanência hospitalar reduzida ${ }^{9,19,20}$. As cirurgias aórticas laparoscópicas tiveram resultados similares ou melhores que os da cirurgia aberta convencional quando comparados parâmetros hemodinâmicos, metabólicos e inflamatórios ${ }^{16}$, assim como em relação ao seguimento em longo prazo ${ }^{7,8,21}$. Elas podem ser realizadas por via totalmente laparoscópica ${ }^{7-9,19}$ ou por minilaparotomia $^{9,10,19,22}$, com resultados semelhantes em longo prazo entre si, já que a única diferença entre as duas está na via de realização das anastomoses ${ }^{19}$.

No entanto, para se atingir tais resultados, é necessário atravessar uma longa curva de aprendizado ${ }^{19,20,23}$. No presente estudo, acreditamos que uma diminuição dos tempos cirúrgicos poderia ser ainda mais significativa se as operações tivessem sido realizadas por um cirurgião principal e mais dois auxiliares, conforme sugerido pela literatura, pois o acúmulo de funções de câmera e auxiliar cirúrgico por um mesmo auxiliar dificultam a realização das anastomoses $^{9,19-21,24}$. Entretanto, mesmo com essas dificuldades técnicas, os tempos cirúrgicos aqui apresentados não diferiram significativamente dos encontrados na literatur $\mathrm{ra}^{8,9,21,23,25}$ 
A técnica videolaparoscópica é mais uma ferramenta minimamente invasiva, viável, segura e eficaz para o tratamento da doença oclusiva aortoilíaca extensa ${ }^{7-9,12,19,21}$. Esta, que nada mais é do que a cirurgia convencional realizada sob visão laparoscópica, tem bons resultados a longo prazo, que se associam à elegância técnica ${ }^{7,8,10,19,20}$, no entando uma longa curva de aprendizado ${ }^{19,20,23,24}$, que decresce com o tempo e à medida que cresce a experiência e vivência com os materiais e com a visão não estereoscópica. Esta técnica pode ser realizada com perfeição por cirurgiões vasculares, sendo que também cirurgiões gerais, ginecologistas e urologistas realizam cirurgias laparoscópicas complexas. O treinamento intensivo, os cursos de laparoscopia e a busca constante de aprimoramento técnico devem ser perseguidos para se obter resultados similares aos da cirurgia convencional.

\section{Agradecimentos}

Os autores agradecem às empresas Johnson \& Johnson - Divisão Ethicon Endo-Surgery, Karl Storz Alemanha e Brasil, pelo apoio e disponibilização de equipamentos e materiais para a realização deste projeto.

\section{Referências}

1. Yoshida RA, Yoshida WB, Maffei FH, et al. Comparative study of evolution and survival of patients with intermittent claudication, with or without limitation for exercises, followed in a specific outpatient setting. J Vasc Bras. 2008; 7:112-22.

2. Lastória S, Maffei FH. Aterosclerose obliterante periférica: epidemiologia, fisiopatologia, quadro clínico e diagnóstico. In: Maffei FH, Lastória S, Yoshida WB, Rollo HA, editores. Doenças vasculares periféricas. $4^{\mathrm{a}}$ ed. Rio de Janeiro: Guanabara-Koogan; 2008. p. 1141-55.

3. Yoshida RA, Yoshida WB, Sobreira M, Silva C. Infrapopliteal angioplasty: the more arteries are treated the better? J Vasc Bras. 2008;7:176-82.

4. Kannel WB, McGee DL. Update on some epidemiologic features of intermittent claudication: the Framingham Study. J Am Geriatr Soc. 1985;33:13-8.

5. Norgren L, Hiatt WR, Dormandy JA, et al. Inter-Society Consensus for the Management of Peripheral Arterial Disease (TASC II). Eur J Vasc Endovasc Surg. 2007;33 Suppl 1:S1-75.

6. Liapis CD, Tzortzis EA. Advances in the management of iliac artery occlusive disease: a short review. Vasc Endovascular Surg. 2004;38:541-5.

7. Kolvenbach R. Total laparoscopic aortic aneurysm surgery. Acta Chir Belg. 2006;106:36-9.

8. Lin JC, Kolvenbach R, Schwierz E, Wassiljew S. Total laparoscopic aortofemoral bypass as a routine procedure for the treatment of aortoiliac occlusive disease. Vascular. 2005;13:80-3.
9. Cau J, Ricco JB, Corpataux JM. Laparoscopic aortic surgery: Techniques and results. J Vasc Surg. 2008;48:37S-44S; discussion $45 \mathrm{~S}$.

10. Yoshida R, Yoshida WB, Kolvenbach R, et al. Cirurgia aortoilíaca videolaparoscópica para tratamento de isquemia crítica de membros: relato do primeiro caso no Brasil. J Vasc Bras. 2009;8:364-70.

11. Dion YM, Thaveau F, Fearn SJ. Current modifications to totally laparoscopic "apron technique". J Vasc Surg. 2003;38:403-6.

12. Coggia M, Bourriez A, Javerliat I, Goeau-Brissonniere O. Totally laparoscopic aortobifemoral bypass: a new and simplified approach. Eur J Vasc Endovasc Surg. 2002;24:274-5.

13. Yoshida WB, Bosco FA, Medeiros FATM, Rollo HA, Dalben IN. Lípides séricos como fator de risco para pacientes com doença arterial periférica. J Vasc Bras. 2003;2:5-12.

14. Junior O, van Bellen B. Transperitoneal and retroperitoneal approach to the abdominal aorta: early results. J Vasc Bras. 2004;3:331-8.

15. Johnson JN, McLoughlin GA, Wake PN, Helsby CR. Comparison of extraperitoneal and transperitoneal methods of aorto-iliac reconstruction. Twenty years experience. J Cardiovasc Surg (Torino). 1986;27:561-4.

16. Kolvenbach R, Deling O, Schwierz E, Landers B. Reducing the operative trauma in aortoiliac reconstructions: a prospective study to evaluate the role of video-assisted vascular surgery. Eur J Vasc Endovasc Surg. 1998;15:483-8.

17. Yoshida WB. Radicais livres na síndrome de isquemia e reperfusão. Cir Vasc Angiol. 1996;12:82-95.

18. Dion YM, Griselli F, Douville Y, Langis P. Early and midterm results of totally laparoscopic surgery for aortoiliac disease: lessons learned. Surg Laparosc Endosc Percutan Tech. 2004;14:328-34.

19. Kolvenbach R, Yoshida R. Cirurgia Aórtica Videolaparoscópica. In: Maffei FHA, Yoshida WB, Lastoria S, Rollo H, Gianini M, Moura R, editores. Doenças vasculares periféricas. Volume 1. 4 ed. Rio de Janeiro: Guanabra-Koogan; 2008. p. 859-86.

20. Yoshida R, Yoshida W, Kolvenbach R, Rollo H, Lorena S. Laparoscopic aortic surgery learning curve: experimental study in pigs. J Vasc Bras. 2008;7:231-8.

21. Coggia M, Javerliat I, Di Centa I, et al. Total laparoscopic versus conventional abdominal aortic aneurysm repair: a case-control study. J Vasc Surg. 2005;42:906-10; discussion 911.

22. Fearn SJ, Thaveau F, Kolvenbach R, Dion YM. Minilaparotomy for aortoiliac aneurysmal disease: experience and review of the literature. Surg Laparosc Endosc Percutan Tech. 2005;15:220-5.

23. Ferrari M, Adami D, Del Corso A, et al. Laparoscopyassisted abdominal aortic aneurysm repair: early and middle-term results of a consecutive series of 122 cases. J Vasc Surg. 2006;43:695-700.

24. Kolvenbach R, Da Silva L, Deling O, Schwierz E. Videoassisted aortic surgery. J Am Coll Surg. 2000;190:451-7.

25. Dion YM, Gracia CR, Estakhri M, et al. Totally laparoscopic aortobifemoral bypass: a review of 10 patients. Surg Laparosc Endosc. 1998;8:165-70. 
Correspondência:

Ricardo de Alvarenga Yoshida

Depto de Cirurgia e Ortopedia

Faculdade de Medicina de Botucatu - UNESP

CEP 18618-970 - Botucatu, SP

Tel.: (14)3811.6269

E-mail: ricardoyoshida@gmail.com

\section{Contribuições dos autores}

Concepção e desenho do estudo: RAY, WBY

Análise e interpretação dos dados: RAY, WBY, RK
Coleta de dados: RAY, WBY, RGJ, FCP, AHGF

Redação do artigo: RAY, WBY

Revisão crítica do texto: RAY, WBY, RK, HAR

Aprovação final do artigo*: RAY, WBY, RGJ, FCP, AHGF, HAR, RK

Análise estatística: N/A

Responsabilidade geral pelo estudo: RAY

Informações sobre financiamento: N/A

* Todos os autores leram e aprovaram a versão final submetida ao J Vasc Bras. 